\title{
BINOMIAL VERSION OF MARKOV MODEL OF FATIGUE LIFE OF COMPOSITE WITH TWO REASONS FOR FAILURE
}

\author{
A.Yu. Paramonova, M.A. Kleinhofs, Yu.M. Paramonov
}

\begin{abstract}
Riga Technical University.E-mail: rauprm@junik.lv
Received 0707 2004, accepted 26072004

Alexandra PARAMONOVA was born on September 24, 1974 in Latvia. Education: Riga Technological University of Civil Aviation; Programming Engineering Diploma (1996), Master of Computer Science (1996). Family status: unmarried. Present Position: Lecturer at Transport and Telecommunication Institute, Lomonosova street, 1, Riga, LV1019, Latvia, +(371)-7255394, rauprm@ junik.lv. Fields of research: Reliability of composite materials; mathematical statistics; object-oriented visual systems design and programming. Publications: sixteen, two textbooks.
\end{abstract}
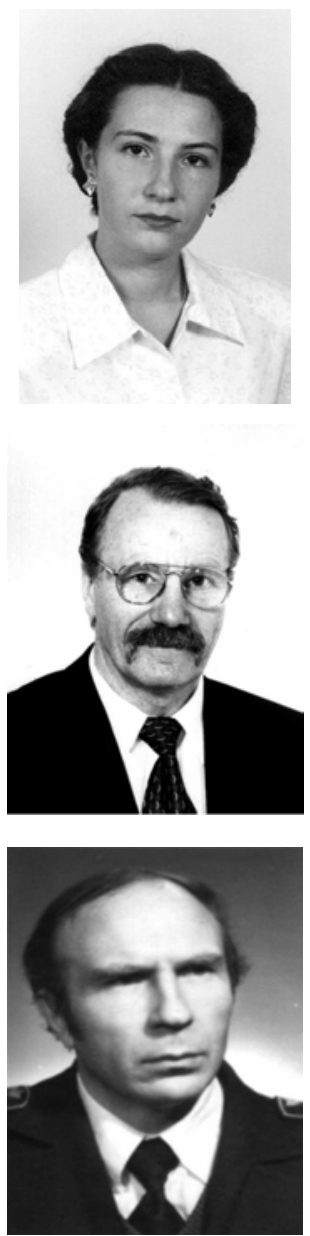

\begin{abstract}
Martinsh KLEINHOFS was born on September 25, 1942 in Latvia. Education: Riga Civil Aviation Engineering Institute; Mechanical Engineering Diploma (1966), Doctor Sc. Eng. Degree (1983), Doctor Habilitus Degree in Eng. (1998). Family status: Married. Present Position: Director of Aviation Institute, Assistant Professor of Aircraft Theory and Structure Department of Aviation Institute of Riga Technical University Lomonosova street, 1 add space, Riga, LV-1019, Latvia, +(371)-7322482, + (371) - 7 089966, Aviation.Institute @ @rtu.lv. Fields of research: Reliability of technical systems; mathematical statistics; loads, structure and strength analysis of transport vehicle; structure and strength of composite materials. Publications: 84 , one certificate of invention, 16 training books.
\end{abstract}

Yuri PARAMONOV was born on March 28, 1938 in Leningrad. Education: Riga Aviation Engineering Military High School, Mechanical Engineering Diploma with goldMedal (1960). Riga Civil Aviation Eng. Institute, Doctor Sc. Eng. Degree (1965). Latvian Academy of Sciences, High Doctor Degree in Technical Cybernetics (1974). Riga Aviation University and Latvian Academy of Science, Doctor Habilitus Degree in Engineering (1993). Family status: Married. Present Position: Professor of Aircraft Theory and Structure Department of Aviation Institute of Riga Technical University, Lomonosova street, 1, add space Riga, LV-1019, Latvia, +(371)-2255394, + (371) - 7089966. Fields of research: Reliability of technical systems; mathematical statistics; loads, structure and strength analysis of transport vehicles; information technology application (development of automated control systems). Member of professional societies: American Statistical Association, Research Board of Advisors of the American Biografical Institute, Participation in 15 international conferences; Publications: 145, including nine monographs and textbooks. Honour awards: Honoured Scientist of Latvian Soviet Socialist Republic (1983); order: Honour Decoration (1971); medals: For Valiant Labour (1970), Labour Veteran (1985); Nomination as an International Man of the Year for $1997 / 98$ by the International Biografical Centre of Cambridge.

\begin{abstract}
A model of fatigue damage accumulation in laminate with two reasons for fatigue failure (the distortion of rigid items and the excessive yielding of the plastic part of the composite (matrix)) is offered. The model is based on the use of the Markov chains theory. It is shown how the corresponding transition probability matrix can be filled and how to calculate the mean time to failure, the variance of this time, and the probability distribution function. This model can be used as a nonlinear regression model for fatigue curve approximation and for fatigue damage accumulation description in program fatigue test. Processing of experimental data (fatigue test of carbon-fiber reinforced laminate for fatigue curve building and for residual fatigue-life investigation in program test with two levels of stress) shows that the model can be used for satisfactory description of the results of these tests. The specific feature of the model considered in this paper is the use of binomial distribution of failure of rigid items and the number of acts of yielding in the plastic part of the laminate.
\end{abstract}

Keywords: strength, yielding, fatigue life, composite, fatigue curve.

\section{Introduction}

The main goal of this paper is the development of ideas, which were discussed in [1, 2, and 3]. We'll consider the same problem - fatigue curve approximation by the use of the Markov Chain theory. In the papers 1, 2 and 3 we considered laminate as a "bundle" of parallel rigid brittle fibers (or strands) stretched between two clamps. But in a new model we also take into account the possibility of permanent plastic deformation of the matrix of the composite. This model of composite specimen loaded by tension is illustrated in Fig 1. 


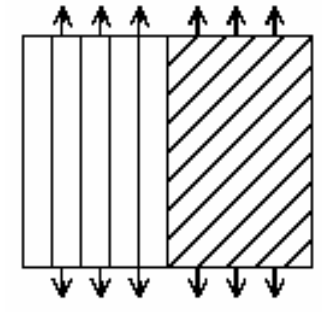

Fig 1. Model of composite specimen

There are two parts of the composite specimen: rigid and plastic. The vertical parallel items of this structure can be considered as a set of rigid brittle items (fibers or strands). Destruction of this part of the composite is the destruction of rigid items (decreasing the number of items which can carry the tension load). The inclined (also parallel) lines symbolically show the possibility of the yielding of the plastic part of the composite. As a result of this yielding, the permanent plastic deformation of the matrix appears. If we consider these two parts of laminate as isolated parts after some yielding, we will see the picture shown in Fig 2. In metal the yielding is connected with some shear strain in some plane. And really this picture is more appropriate to for metal than for composite. But we'll use this model for composite only symbolically just because it is very clear and simple. We'll make the assumption that there is some "set of weakest planes," with a limited number of such "planes" in which shear can appear. And we assume that if in some plane shear took place, then another can take place only in some other plane (but in the finite "set of planes").

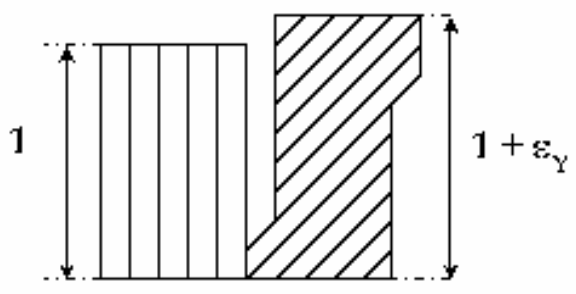

Fig 2. Model of yielding

As a result of yielding, the length of a plastic part increases. The $\varepsilon_{Y 1}$-value is the value of permanent deformation after one act of yielding (one shear in some plane) takes place. We'll make the assumption that this unit-size of permanent deformation is a constant, and it is some parameter of the model considered. During fatigue loading, the accumulation of permanent plastic deformation takes place. If the number of acts of yielding exceeds some critical limit (another parameter of the model), then a failure of the composite matrix takes place and the failure of the composite matrix is considered a failure of the composite as a whole.

Another reason for failure of composite is the destruction of rigid items. If a rigid elastic part and a plastic part continue to be together, after elimination of an external load, internal stresses appear. Tension appears in the rigid part and compression in the plastic part. As a consequence of this phenomenon the stress of the rigid part in the next cycle of the cycling load increases. Probability of failure of any rigid item also increases. The stresses in the plastic part decrease, but yielding can appear again, although with some smaller probability and in an other plane.

So we consider two reasons of destruction: destruction of the rigid elastic part and excessive yielding of the plastic part.

The general description of the model with elastic and plastic parts is not new (but some specific details of accepted assumptions are new). The main purpose of this paper is to give the most simple mathematical description of this model which should be enough to get a satisfactory quantitative description of the connection of the static strength distribution parameter with the parameters of fatigue curve and the parameter of fatigue life distribution in program fatigue loading. By processing experimental data, it is shown that the model that is presented gives a satisfactory description of fatigue curve and can be applied to the problem to forecast fatigue damage accumulation in program cycling loading. More precisely, it is shown that this model gives a reasonable description of experimental data for program loading with a one step change in mode of loading. The specific feature of this model is the use of binomial distribution of the number of failures of rigid items and the number of acts of yielding in one transition of the Markov Chain.

The mathematical model will be given in the second part of the paper. An application for fatigue damage accumulation for program loading is considered in the third part. The numerical example is considered in the last part.

\section{Mathematical model}

Really we should consider the Markov Chain in twodimension state space because there are two reasons for specimen failure: as a consequence of failures of rigid items and as a consequence of an excessive number of acts of yielding. The first dimension is the number of failed rigid items, and the second one is the number of acts of yielding. But as we will see later, the number of states (in processing of real experimental data) is not too large in every dimension. So by a corresponding renumbering of the states, we will use a description of ordinary one- dimension state space. We will consider the transition probability matrix as a complex of blocks. The number of blocks is equal to one plus the critical number of acts of yielding corresponding to the yield-type destruction. The number of states inside every block is equal to one plus the critical number of failures of rigid items corresponding to the brittle-type failure of rigid items.

For the simplest example, let the destruction of specimens take place if :

(1) there is a failure of two rigid items (event A)

or

(2) there are two acts of yielding (event B)

or

(3) both events A and B take place. 
The corresponding transition probability matrix is symbolically described in Table 1 . In this table together with the usual indexes of matrix items, $i$ and $j$, the "local" indexes, $i_{Y}, i_{R}, j_{Y}, j_{R}$, are shown also. The number of acts of yielding equals $\left(i_{Y}-1\right)$ or $\left(j_{Y}-1\right)$. The number of failed rigid items is equal to $\left(i_{R},-1\right)$ or $\left(j_{R},-1\right)$, where $i$ corresponds to the initial state and $j$ to the final state of one transition. In general $r_{R}$ is the number of failures of rigid items, and $r_{Y}$ is the number of acts of yielding corresponding to the failure of specimens.

The interrelations between these indexes are defined by the formulae

$$
\begin{aligned}
& i=\left(r_{R}+1\right) *\left(i_{Y}-1\right)+i_{R} ; \\
& j=\left(r_{R}+1\right) *\left(j_{Y}-1\right)+j_{R} .
\end{aligned}
$$

In Table 1 for $r_{R}=2, i, j=1,2, \ldots, 9$ correspond to nine states of the Markov Chain (three possible values of failure of rigid items $(0,1,2)$ to multiply by three possible values of acts of yielding $(0,1,2))$. Probabilities $p_{R 0}, p_{R l}, \ldots$ denote probabilities of failure of $0,1, \ldots$ rigid items. Probabilities $p_{Y 0}, p_{Y l}, \ldots$ denote probabilities of 0,1 , ... acts of yielding. In Table 1 these notations have only a a symbolical sense, but really these probabilities depend on the state of the Markov Chain. The corresponding formulae are given later. In the chain we have five absorbing states:

$\mathrm{S}_{3}$ is the failure of two rigid items (FR) without yielding,

$\mathrm{S}_{6}$ is FR after one act of yielding,
$\mathrm{S}_{7}, \mathrm{~S}_{8}$ are distortion after two acts of yielding when the number of failed rigid items can be equal to 0,1 .

State $S_{9}$ is reached when the number of failed rigid items is equal to $r_{R}$ and the number of acts of yielding is equal to $r_{Y}$ simultaneously.

In this paper, it is supposed that the number of failures of rigid items in one transition of the Markov Chain has binomial distribution. If there are $n_{R}$ still efficient rigid items then the probability of the event "number of failures of rigid items is equal to $k_{R}$ " is defined by the formula

$$
\begin{aligned}
& p_{R}(i, j)=\left(\begin{array}{l}
n_{R} \\
k_{R}
\end{array}\right)\left(F_{R}\left(S_{R}\left(i_{R}, i_{Y}\right)\right)\right)^{k_{R}} * \\
& \left(1-F_{R}\left(S_{R}\left(i_{R}, i_{Y}\right)\right)\right)^{n_{R}-k_{R}}
\end{aligned}
$$

where $n_{R}=r_{R}-i_{R}$,

$k_{R}=j_{R}-i_{R}$.

for $0 \leq k_{R} \leq n_{R}, \quad 1 \leq n_{R} \leq\left(r_{R}-1\right)$.

$F_{R}($.$) is the distribution function of the strength of$ still efficient rigid items.

$S_{R}\left(i_{R}, i_{Y}\right)$ is the stress in the rigid part in ith state (see formulae (1)).

\begin{tabular}{|c|c|c|c|c|c|c|c|c|c|c|c|}
\hline & & $\mathbf{j}_{\mathbf{Y}}$ & \multicolumn{3}{|c|}{1} & \multicolumn{3}{|c|}{2} & \multicolumn{3}{|c|}{3} \\
\hline & & $\mathbf{j}_{\mathbf{R}}$ & 1 & 2 & 3 & 1 & 2 & 3 & 1 & 2 & 3 \\
\hline $\mathbf{i}_{\mathbf{Y}}$ & $\mathbf{i}_{\mathbf{R}}$ & $\mathbf{i} \backslash \mathbf{j}$ & 1 & 2 & 3 & 4 & 5 & 6 & 7 & 8 & 9 \\
\hline \multirow{3}{*}{1} & 1 & 1 & $\mathrm{p}_{\mathrm{R} 0} \mathrm{p}_{\mathrm{Y} 0}$ & $\mathrm{p}_{\mathrm{R} 1} \mathrm{p}_{\mathrm{Y} 0}$ & $\mathrm{p}_{\mathrm{R} 2} \mathrm{p}_{\mathrm{Y} 0}$ & $\mathrm{p}_{\mathrm{R} 0} \mathrm{p}_{\mathrm{Y} 1}$ & $\mathrm{p}_{\mathrm{R} 1} \mathrm{p}_{\mathrm{Y} 1}$ & $\mathrm{p}_{\mathrm{R} 2} \mathrm{p}_{\mathrm{Y} 1}$ & $\mathrm{p}_{\mathrm{R} 0} \mathrm{p}_{\mathrm{Y} 2}$ & $\mathrm{p}_{\mathrm{R} 1} \mathrm{p}_{\mathrm{Y} 2}$ & $\mathrm{p}_{\mathrm{R} 2} \mathrm{p}_{\mathrm{Y} 2}$ \\
\hline & 2 & 2 & 0 & $\mathrm{p}_{\mathrm{R} 0} \mathrm{p}_{\mathrm{Y} 0}$ & $\mathrm{p}_{\mathrm{R} 1} \mathrm{p}_{\mathrm{Y} 0}$ & 0 & $\mathrm{p}_{\mathrm{R} 0} \mathrm{p}_{\mathrm{Y} 1}$ & $\mathrm{p}_{\mathrm{R} 1} \mathrm{p}_{\mathrm{Y} 1}$ & 0 & $\mathrm{p}_{\mathrm{R} 0} \mathrm{p}_{\mathrm{Y} 2}$ & $\mathrm{p}_{\mathrm{R} 1} \mathrm{p}_{\mathrm{Y} 2}$ \\
\hline & 3 & 3 & 0 & 0 & 1 & 0 & 0 & 0 & 0 & 0 & 0 \\
\hline \multirow{3}{*}{2} & 1 & 4 & 0 & 0 & 0 & $\mathrm{p}_{\mathrm{R} 0} \mathrm{p}_{\mathrm{Y} 0}$ & $\mathrm{p}_{\mathrm{R} 1} \mathrm{p}_{\mathrm{Y} 0}$ & $\mathrm{p}_{\mathrm{R} 2} \mathrm{p}_{\mathrm{Y} 0}$ & $\mathrm{p}_{\mathrm{R} 0} \mathrm{p}_{\mathrm{Y} 1}$ & $\mathrm{p}_{\mathrm{R} 1} \mathrm{p}_{\mathrm{Y} 1}$ & $\mathrm{p}_{\mathrm{R} 2} \mathrm{p}_{\mathrm{Y} 1}$ \\
\hline & 2 & 5 & 0 & 0 & 0 & 0 & $\mathrm{p}_{\mathrm{R} 0} \mathrm{p}_{\mathrm{Y} 0}$ & $\mathrm{p}_{\mathrm{R} 1} \mathrm{p}_{\mathrm{Y} 0}$ & 0 & $\mathrm{p}_{\mathrm{R} 0} \mathrm{p}_{\mathrm{Y} 1}$ & $\mathrm{p}_{\mathrm{R} 1} \mathrm{p}_{\mathrm{Y} 1}$ \\
\hline & 3 & 6 & 0 & 0 & 0 & 0 & 0 & 1 & 0 & 0 & 0 \\
\hline \multirow{3}{*}{3} & 1 & 7 & 0 & 0 & 0 & 0 & 0 & 0 & 1 & 0 & 0 \\
\hline & 2 & 8 & 0 & 0 & 0 & 0 & 0 & 0 & 0 & 1 & 0 \\
\hline & 3 & 9 & 0 & 0 & 0 & 0 & 0 & 0 & 0 & 0 & 1 \\
\hline
\end{tabular}

Similarly the probability that the number of acts of yielding is equal to $k_{Y}$ is defined by a similar formula

Table 1. Transition probabilities matrix 


$$
\begin{aligned}
& p_{Y}(i, j)=\left(\begin{array}{c}
n_{Y} \\
k_{Y}
\end{array}\right)\left(F_{Y}\left(S_{Y}\left(i_{R}, i_{Y}\right)\right)\right)^{k_{Y}} * \\
& \left(1-F_{Y}\left(S_{Y}\left(i_{R}, i_{Y}\right)\right)\right)^{n_{Y}-k_{Y}}
\end{aligned}
$$

where $n_{Y}=r_{Y}-i_{Y}$,

$k_{Y}=j_{Y}-i_{Y}$,

for $0 \leq k_{Y} \leq n_{Y}, \quad 1 \leq n_{Y} \leq\left(r_{Y}-1\right)$,

$F_{Y}($.$) is the distribution function of the stress of$ yielding,

$j_{Y}$ is the number of acts of yielding that have already taken place,

$S_{Y}\left(i_{R}, i_{Y}\right)$ is the stress in a plastic part when the number of acts of yielding is equal to $\left(i_{Y}-1\right)$ but the number of failed rigid items is equal to $\left(i_{R},-1\right)$.

In this paper we will remain in the framework of assumptions that have already been mentioned. The not too bad final result of processing of experimental data can be considered a reasonable excuse for these assumptions.

Local stress itself is the function of applied initial stress, and it is a function of the number of failures of rigid items and of number of acts of yielding that have already taken place. Let the initial cross-section of considered (weakest) critical volume be

$$
f=f_{R}+f_{Y},
$$

where $f_{R}, f_{Y}$ are the cross-section of rigid and yielding parts of critical volume correspondingly.

If the failure of $i$ rigid items takes place, the crosssection of this part decreases:

$$
f_{R i}=f_{R} \cdot\left(1-i / r_{R}\right) .
$$

The cross-section of the yielding part does not change, but its length changes as a function of the number of acts of yielding.

If both rigid and plastic parts are working within the limits of elasticity, we have two equations for corresponding stress calculation:

$$
\left\{\begin{array}{l}
S_{R} \cdot f_{R}+S_{Y} \cdot f_{Y}=S \cdot f \\
\frac{S_{R}}{E_{R}}=\frac{S_{Y}}{E_{Y}}
\end{array}\right.
$$

where $S$ is mean stress, $E$ is Young's modulus, and $R$ and $Y$ are subscripts of the rigid and yielding parts correspondingly. The first equation is equation of equilibrium; the second is equality of strains on both parts. If the lengths of both parts are equal, we have the following solution of this equation system:

$$
\begin{aligned}
& S_{R}=S \cdot f /\left(f_{R}+f_{Y} \cdot E_{Y} / E_{R}\right), \\
& S_{Y}=S \cdot f /\left(f_{Y}+f_{R} \cdot E_{R} / E_{Y}\right) .
\end{aligned}
$$

But if we have some yielding of the plastic part, and it new length becomes equal to $I_{Y}=\left(1+\varepsilon_{Y}\right)$ instead of initial length (initially $I_{Y}=1$ ), we should take into account the residual stress that appears in both parts after outside load is eliminated. This residual stress can be found as a solution of the equation system:

$$
\left\{\begin{array}{l}
\Delta S_{R} \cdot f_{R}=\Delta S_{Y} \cdot f_{Y}, \\
1+\frac{\Delta S_{R}}{E_{R}}=\left(1+\varepsilon_{Y}\right)\left(1-\frac{\Delta S_{Y}}{E_{Y}}\right) .
\end{array}\right.
$$

Here again the first equation is the equation of equilibrium, and the second is the equation of length equality. The solution of this system (in limit of elasticity) is defined the formulae

$$
\begin{aligned}
\Delta S_{R} & =E_{R} \cdot \varepsilon_{Y} /\left(1+\left(1+\varepsilon_{Y}\right) f_{R} E_{R} / f_{Y} E_{Y}\right), \\
\Delta S_{Y} & =E_{Y} \cdot \varepsilon_{Y} /\left(1+\varepsilon_{Y}+f_{Y} E_{Y} / f_{R} E_{R}\right) .
\end{aligned}
$$

We make the additional assumption that in increasing the length of that rigid part the value of $\varepsilon_{Y}$ is proportional to the number of acts of yielding $\left(i_{Y}-1\right)$ :

$$
\varepsilon_{Y}=\varepsilon_{Y 1} \cdot\left(i_{Y}-1\right), i_{Y}=1, \ldots, r_{Y} .
$$

The value of $\varepsilon_{Y 1}$ is considered the parameter of the model.

By renumbering the states of the matrix of transition probabilities can be transformed to the following structure,

$$
P=\left[\begin{array}{ll}
Q & R \\
0 & I
\end{array}\right],
$$

where $\mathrm{Q}$ is the matrix corresponding to transition inside the set of transient states, matrix $\mathrm{R}$ controls the transitions from transient states to absorbing states, matrix $\mathrm{I}$ is an identity matrix, and 0 is a matrix of zeros.

As is known, the vector of mean step numbers to absorption from different transient states is defined by the formula

$\tau=N \cdot \xi$, and

where $N=(I-Q)^{-1}$,

$\xi$ is a column vector of units.

The vector of variances of the corresponding times is defined by the formula

$$
\tau_{2}=(2 N-I) \tau-\tau_{s q},
$$

where $\tau_{s q}(i)=(\tau(i))^{2}, i \in I_{T}$,

$I_{T}$ is a set of indexes of transient states. The matrix of absorption probabilities is defined by the formulas

$$
B=\left\{B_{i j}\right\}=N R,
$$


where $B_{i j}$ is the probability of absorption in $S_{j}$ absorbing state if the initial transient state is $S_{i}$ (renumbering of the states should be taken into account!). Vector of probability functions of times to absorption from different initial transient states is defined the formula

$$
\left\{F_{T}(t)\right\}=P^{t} b
$$

where $b$ is a column vector of the type $(0, \ldots$ $0, \ldots, 1, \ldots, 1)$ '. Here the ordered number of units is equal to the ordered number of absorbing states of matrix $P$ (renumbering of the states should be taken into account again!).

The probability distribution function of time to failure from the initial state $S_{l}$ is defined by the formula

$$
F_{T}(t)=a P^{t} b,
$$

where $a$ is a row vector of type $(1,0,0, \ldots 0)$.

\section{Application for program loading}

This model can very easily be used for fatigue life calculation for program fatigue test. For any arbitrary stress cycle sequence $\left\{S_{1}, S_{2}, S_{3}, \ldots\right\}$, the probability distribution function of time to failure from an initial state is defined by the formula

$$
F_{T}(t)=a\left(\prod_{i=1}^{t} P_{i}\right) b
$$

where matrix $P_{i}$ is the matrix of transition probabilities corresponding to the stress $S_{i}$ and $i=1,2,3, \ldots ; a, b$ are the same as in previous formula. If stress changes in accordance with the block program which is shown in Fig 3 ,

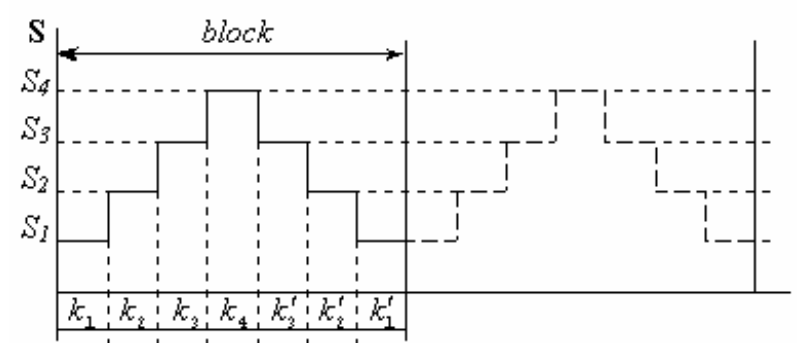

Fig 3. Example of block program

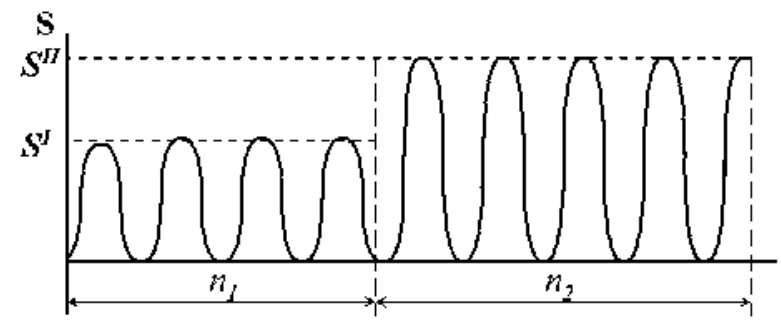

Fig 4. Program of two stress levels then the probability transition matrix corresponding to one block will be defined by the formula

$$
P_{B}=P_{S 1}^{k_{1}} P_{S 2}^{k_{2}} P_{S 3}^{k_{3}} P_{S 4}^{k_{4}} P_{S 3}^{k_{3}^{\prime}} P_{S 2}^{k_{2}^{\prime}} P_{S 1}^{k_{1}^{\prime}},
$$

where $P_{S i}$ is transition probability matrix, corresponding to stress $S_{i}, i=1, \ldots, 4$. By the use of $P_{B}$ instead of matrix $P$ we can easily calculate the fatigue life distribution function $F_{T}(t)=a P^{t} b$ and its numerical characteristics $\tau$ and $\tau_{2}$ if we use the block as a time unit.

A special case of loading is shown in Fig 4.

For this program the distribution function of time to failure is defined by the formula

$$
F_{T}(t)=\left\{\begin{array}{l}
a\left(P_{S^{i}}^{t}\right) b, \text { if } \mathrm{t} \leq \mathrm{n}_{1}, \\
a\left(P_{S^{I}}^{n_{1}} P_{S^{i i}}^{t-n_{1}}\right) b, \text { if } \mathrm{t}>\mathrm{n}_{1} .
\end{array}\right.
$$

The conditional distribution function of residual fatigue life, $T_{2}$ (provided that there was not failure of the specimen in the first stage test after $n_{1}$ cycles with $S=S^{I}$ ), as usually, is defined by formula

$$
F_{T_{2}}(t)=\frac{F_{T}\left(n_{1}+t\right)-F_{T}\left(n_{1}\right)}{1-F_{T}\left(n_{1}\right)} .
$$

In this paper we will limit ourselves by checking the forecast of the expected value of $n_{2}$ if the fatigue curve is known and $n_{1}$ is known also. The calculation of the expected value of $n_{2}$ is similar to the calculation of $\mathrm{E}(\mathrm{T})$, and $\mathrm{V}(\mathrm{T})$ for the case without stress change, but we should take into account that the order number of initial state at the end of $n_{l}$ cycles with stress $S=S^{I}$ is the random variable with distribution

$$
\pi^{I I}=(1,0,0 \ldots) P_{S^{I}}^{n_{1}} .
$$

Then the expected value of cycles to failure in the second stage with $S=S^{I I}$ is defined by the formula

$$
E\left(T_{2}\right)=\pi^{I I} \cdot \tau^{I I} .
$$

The variance of this time is defined by the formula

$$
\begin{aligned}
& V\left(T_{2}\right)=E\left(T_{2}^{2}\right)-(E(T))^{2}= \\
& \pi^{I I} \cdot\left(\tau_{2}^{I I}+\tau_{s q}^{I I}\right)-\left(\pi^{I I} \tau^{I I}\right)^{2}
\end{aligned}
$$




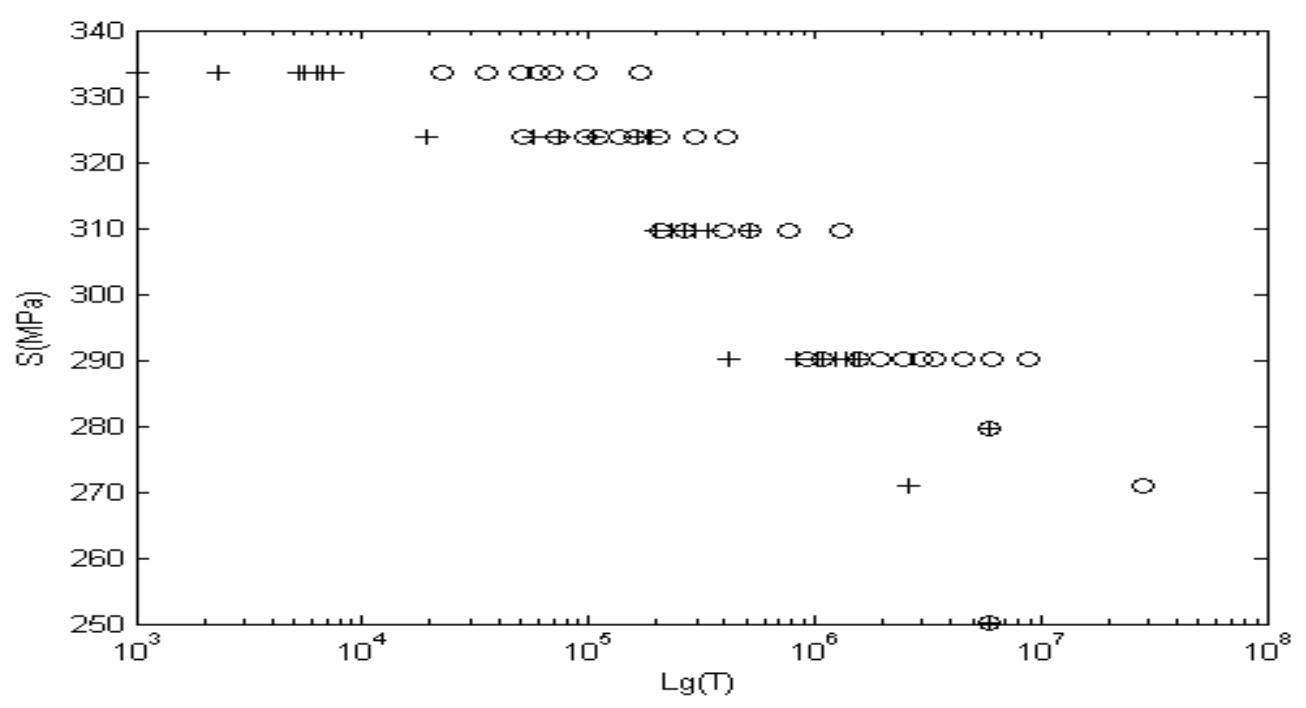

Fig 5. Fatigue curves (experimental: "+”; calculation result: “o”)

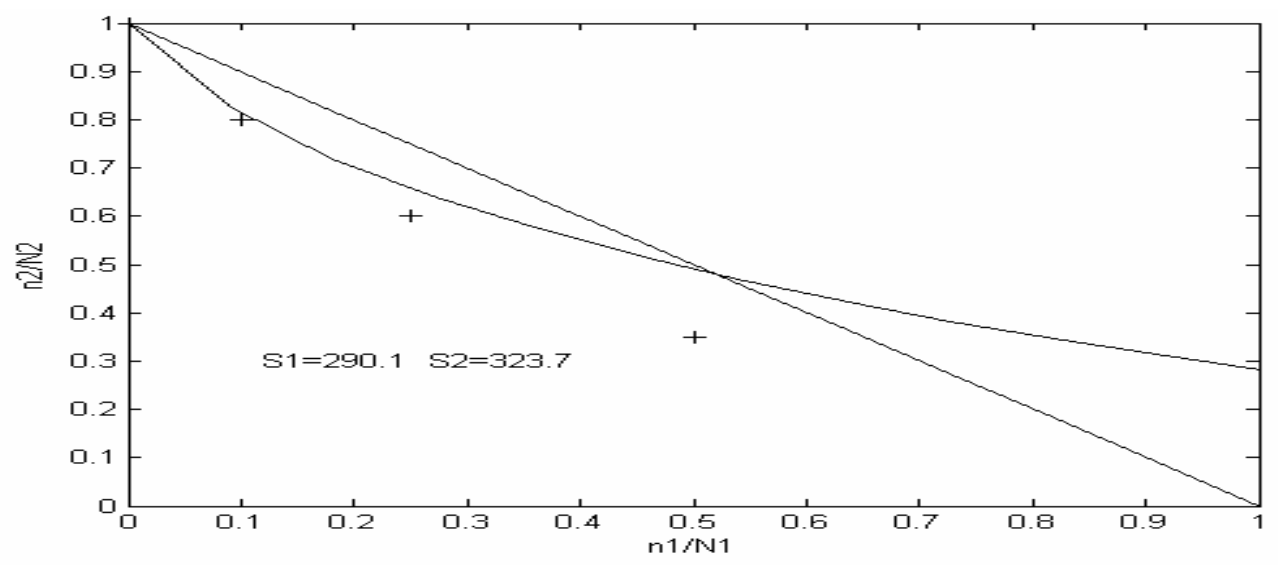

Fig 6. Relative residual fatigue life as function of relative damage in first stage of program loading for the case $S^{I}<S^{I I}$

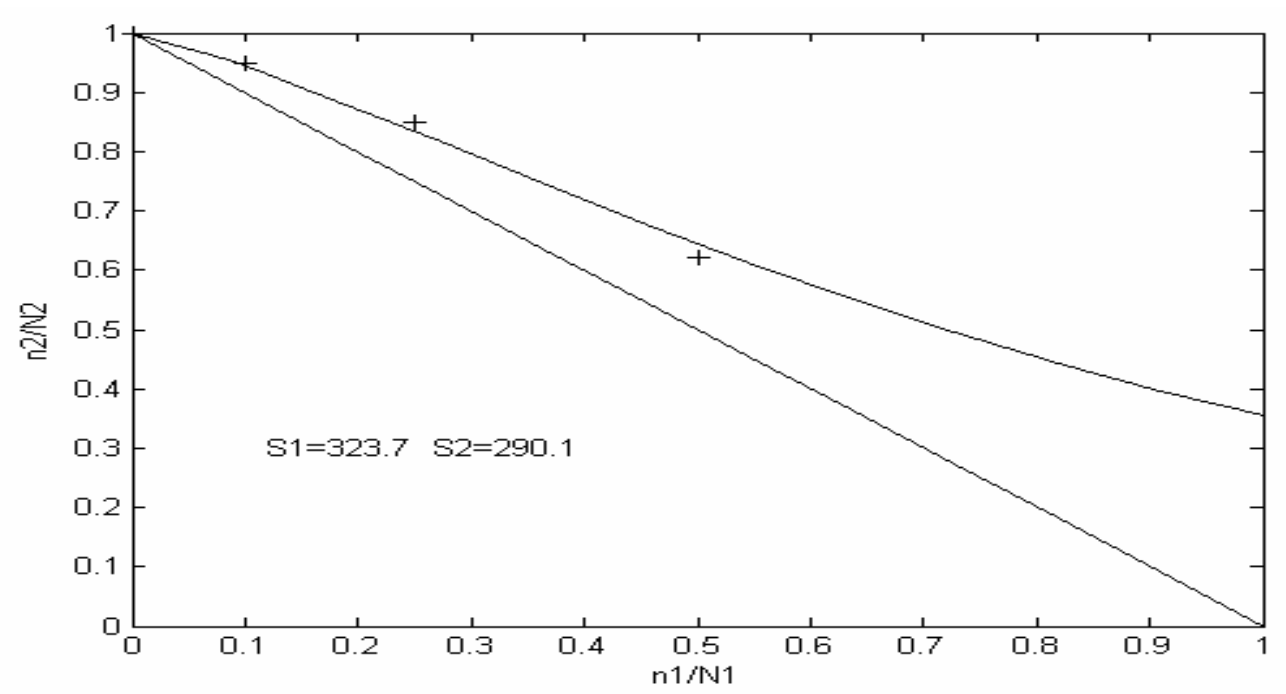

Fig 7. Relative residual fatigue life, $n_{2} / N_{2}$, as function of relative damage $n_{1} / N_{1}$ in first stage of program loading for the case $S^{I}>S^{I I}$ 


\section{Processing of experimental data}

Experimental data was obtained by Kleinhofs, who tested carbon-fiber specimens to get the fatigue curve and to study the residual fatigue lives in step-up (see Fig 4) and step-down program [4]. The results of processing these data are shown in Figures 5, 6, and 7. In Fig 5 experimental data for a fatigue curve are shown by "+". The result of calculations of order statistics (corresponding to the size of the experimental sample size) for every stress level for which the test was made is shown by "o". At the lowest stress level, a limitation of test time (censoring) took place.

Dependence of the relative residual fatigue life $n_{2} / N_{2}$ on the relative damage $n_{l} / N_{l}$ of the first step of program cyclic loading is shown in Fig 6 and Fig 7.

Here the results of calculations are shown by curved lines, but experimental data are again shown by "+".

We see that at chosen parameters, just as in the test, at small $n_{l} / N_{l}$ the calculation gives

$$
\begin{aligned}
& \frac{n_{1}}{N_{1}}+\frac{n_{2}}{N_{2}}>1 \text { if } S^{I}>S^{I I} \\
& \text { and } \frac{n_{1}}{N_{1}}+\frac{n_{2}}{N_{2}}<1 \text { if } S^{I}<S^{I I} .
\end{aligned}
$$

In order to understand the behavior of these curves, we should take into account the failure of some specimens already in the first stage of program cyclic loading Only the strongest specimens can be used for loading by stress $S^{I I}$. It is worth mentioning, in particular, that usually in corresponding publications in this type of figures $n_{2} / N_{2}=0$ when $n_{1} / N_{1}=1$.

But this can be true only if we do not take into account the scatter of fatigue life. Actually, at $n_{1} / N_{1}=1$ approximately one half of the specimens are not destroyed. So corresponding mean residual life is not equal to zero! And it is can be seen in the Figures 6 and 7 .

\section{Conclusion}

The suggested model of fatigue damage accumulation in composite material allows a reasonably good approximation of

(1) fatigue curve,

(2) distribution function of fatigue life (for the case of loading with constant stress cycle parameter and in a case of program loading).

The specific feature of this model is that it takes into account not only the failure of rigid items, but also the yielding of the matrix of the composite. For a one step stress change program of cyclic loading, it allows one to explain the deviation of the sum $\left(n_{1} / N_{1}+n_{2} / N_{2}\right)$ from the unit (from Miner's Rule) in the same direction as in real test..

\section{References}

1. Paramonova A.Yu., Kleinhofs M.A., Paramonov Yu.M. Markov chains theory use for fatigue curve of composite material approximation // Aviation. - V.: Technika, 2002. - No 6. - P. 103 - 108.

2. Paramonova A.Yu., Kleinhofs M.A., Paramonov Yu.M. 2003. The estimation of parameters of fatigue curve of composite material // Aviation. - V.: Technika, 2003. - Vol VII, No 6. - P. 28 - 37.

3. Paramonova A.Yu., Kleinhofs M.A., Paramonov Yu.M. The use of Markov chains theory for approximation of fatigue curve of composite material $/ / 3^{\text {rd }}$ International Conference on Mathematical Methods in Reliability, 2002 Norway. - 2002. - P. 509-512.

4. Клейнхофс М.А. Исследование статической и усталостной прочности композитных материалов, используемых в конструкции летательных аппаратов: Дис. ...канд. тех. наук. - Рига, 1983. 